\title{
Peculiar Distribution of Tumorous Xanthomas in an Adult Case of Erdheim-Chester Disease Complicated by Atopic Dermatitis
}

\author{
Yukako Murakami ${ }^{a}$ Mari Wataya-Kaneda ${ }^{a}$ Mika Terao ${ }^{a}$ \\ Hiroaki Azukizawa ${ }^{a}$ Hiroyuki Murota $^{a}$ Yukiko Nakata $^{\mathrm{b}}$ \\ Ichiro Katayama ${ }^{a}$
}

Departments of a Dermatology and ${ }^{b}$ Clinical Laboratory Diagnostics, Osaka University

Graduate School of Medicine, Osaka, Japan

\section{Key Words}

Erdheim-Chester disease $\cdot$ Hand-Schüller-Christian disease $\cdot$ Xanthoma - Atopic

dermatitis - Macrophage - CD68 - CD163 - Thymus- and activation-regulated chemokine

\begin{abstract}
Erdheim-Chester disease is a rare non-Langerhans form of histiocytosis with multiple organ involvement. Approximately $20 \%$ of patients have xanthoma-like lesions, usually on the eyelids. We report a case of Erdheim-Chester disease in a 32-year-old male who showed peculiar xanthomatous skin lesions and also had atopic dermatitis. His skin manifestations included ring-like yellowish tumors on his periorbital regions, rope necklace-like tumors on his neck, and spindle-shaped tumors on his right preauricular region and cubital fossas. He also had exophthalmos and diabetes insipidus. Chronic eczematous lesions were present on the flexor aspect of his extremities, and his serum eosinophil numbers and immunoglobulin E levels were elevated. A histological examination of his right neck tumor showed foamy macrophages and touton-type giant cells, which were positive for CD68 and CD163 and negative for S-100 and CD1a. We suggest that the complication of atopic dermatitis may have contributed to the uncommon clinical features in this case.
\end{abstract}

\section{Case Report}

A 32-year-old male presented with xanthomatous skin lesions, exophthalmos, and diabetes insipidus. His left retro-orbital mass was partially removed in childhood at another hospital, and he had been diagnosed with Hand-Schüller-Christian disease (HSCD). His past medical history included hyperlipidemia, type 2 diabetes mellitus, hypertension, and hyperuricemia, which were managed with 
diet and medication (nateglinide, candesartan cilexetil, and allopurinol). Cutaneous examination revealed ring-like yellowish tumors on his periorbital regions (fig. 1a), rope necklace-like tumors on his neck (fig. 1b, c), and spindle-shaped tumors on his right preauricular region and cubital fossas (fig. 1d). Histological examination of his right neck tumor showed foamy macrophages and touton-type giant cells (fig. 2a). Immunohistochemical staining revealed that the cells were positive for CD68 (fig. 2b) and CD163 (fig. 2c) and negative for S-100 and CD1a. X-rays of the long bones of the upper and lower extremities showed no apparent osteosclerotic or osteolytic changes. Exophthalmos, diabetes insipidus, and the pathological findings confirmed the diagnosis of Erdheim-Chester disease (ECD; table 1). The patient had also been diagnosed with atopic dermatitis in childhood. He demonstrated diffuse facial erythema, pruritic chronic eczematous lesions on the trunk and flexor aspect of the extremities (fig. 1d), and multiple prurigo of the extremities. Laboratory findings related to atopic dermatitis were white

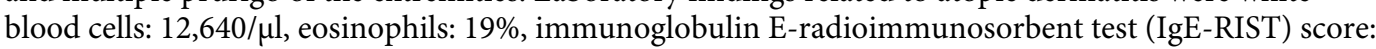
38,500 IU/ml, IgE radioallergosorbent test (IgE-RAST) score of Dermatophagoides farinae: $99.9 \mathrm{IU}$, and thymus- and activation-regulated chemokine (TARC): 47,980 pg/ml. Therefore, our patient was diagnosed with ECD complicated by atopic dermatitis.

\section{Discussion}

ECD is a rare non-Langerhans form of histiocytosis with multiple organ involvement. Involvement of the long bones is observed in $86 \%$ of patients, and a typical manifestation is bilateral symmetric sclerosis of the metaphyseal region of the long bones of the lower extremities $[1,2]$. Approximately one half of all cases have extraskeletal manifestations, including effects on the hypothalamus-pituitary axis, lungs, heart, retroperitoneum, skin, liver, kidneys, spleen, and orbit. Skin involvement is seen in approximately $20 \%$ of patients, who frequently present with xanthoma-like lesions that usually manifest on the eyelids and occasionally on the trunk and submammary area [3].

Here, we report a case of ECD with a peculiar distribution of tumorous xanthomas and without bone involvement. The patient was initially diagnosed in childhood with HSCD, one of the syndromes of Langerhans cell histiocytosis (LCH), based on the histological findings of his left retro-orbital mass. It is sometimes difficult to distinguish ECD from HSCD because they have certain clinical findings in common such as exophthalmos, diabetes insipidus, and radiological findings of osseous lesions. Osteolysis of the scalp bones is typical in HSCD, whereas osteosclerosis of the long bones is the characteristic presentation of ECD. However, osteosclerosis in ECD is sometimes difficult to identify, and osteolytic lesions are found in approximately one third of patients with ECD [4]. ECD commonly occurs in adulthood, whereas HSCD occurs in infancy. The mortality rate for ECD is $57 \%$ and for HSCD it is $30 \%$ [5] (table 1).

Immunohistochemical analyses are useful for distinguishing between these two diseases. The histiocytes in the skin involve two major cell lineages, macrophages and Langerhans cells, which are derived from monocytes [6]. Pathogenic macrophages are observed in ECD. In contrast, Langerhans cells are characteristic of HSCD. The histiocytes in ECD are immunoreactive for CD68 and CD163, but not for S-100 or CD1a. The pattern is reversed in histiocytes in HSCD (table 1). Because we were not able to obtain detailed histological information on the left retro-orbital mass removed in childhood, we could not conclude that this was a case of HSCD in childhood that converted to ECD in adulthood, or a case of ECD that showed an HSCD-like manifestation in childhood. Previously, it was suggested but not definitively established that ECD may represent a spectrum of $\mathrm{LCH}[7,8]$. However, it was recently suggested 
that ECD is a unique disease entity and LCH-like findings may be within the spectrum of ECD $[9,10]$. Further examination of cases and research are needed to explore this issue.

It is interesting that tumorous xanthomas were present mainly in sites predisposed to atopic dermatitis such as the neck skin and flexor aspect of the extremities. Although some cases show nodular xanthomatous masses of the neck [11], rope necklace-like xanthomas and bulging xanthomas on the flexor aspect of the extremities as observed in the present case are rare. We suggest that the complication of atopic dermatitis modified the clinical presentation in this case. Activation of fibroblasts due to scratching behavior evoked by itching due to atopic dermatitis [12] may have resulted in tumorous xanthomas.

In conclusion, we reported a case of ECD with a peculiar distribution of tumorous xanthomas. We suggested that the complication of atopic dermatitis played a role in the development of the uncommon clinical features of our case.

Table 1. Hand-Schüller-Christian disease and Erdheim-Chester disease

\begin{tabular}{llllllll}
\hline & Onset & Clinical sign & Mortality & $\begin{array}{l}\text { Proliferated } \\
\text { histiocyte }\end{array}$ & S-100 CD1a CD68 CD163 \\
\hline HSCD & Infant & DI, exophthalmos, osteolysis of the scalp bone & $30 \%$ & Langerhans cell & + & + & - \\
ECD & Adult & DI, exophthalmos, osteosclerosis of long bones \\
& & (sometimes osteolysis) & $57 \%$ & Macrophage & - & - & + \\
\hline
\end{tabular}

DI = Diabetes insipidus; HSCD = Hand-Schüller-Christian disease; ECD = Erdheim-Chester disease. 


\begin{tabular}{r|l|l|l}
$\begin{array}{c}\text { Case Reports in } \\
\text { Dermatology }\end{array}$ & $\begin{array}{l}\text { Case Rep Dermatol 2011;3:107-112 } \\
\text { DOI: } 10.1159 / 000328797\end{array}$ & $\begin{array}{l}\text { Published online: } \\
\text { May 17, 2011 }\end{array}$ & $\begin{array}{l}\text { I 2011 S. Karger AG, Basel } \\
\text { ISSN 1662-6567 } \\
\text { www.karger.com/cde }\end{array}$ \\
\hline
\end{tabular}
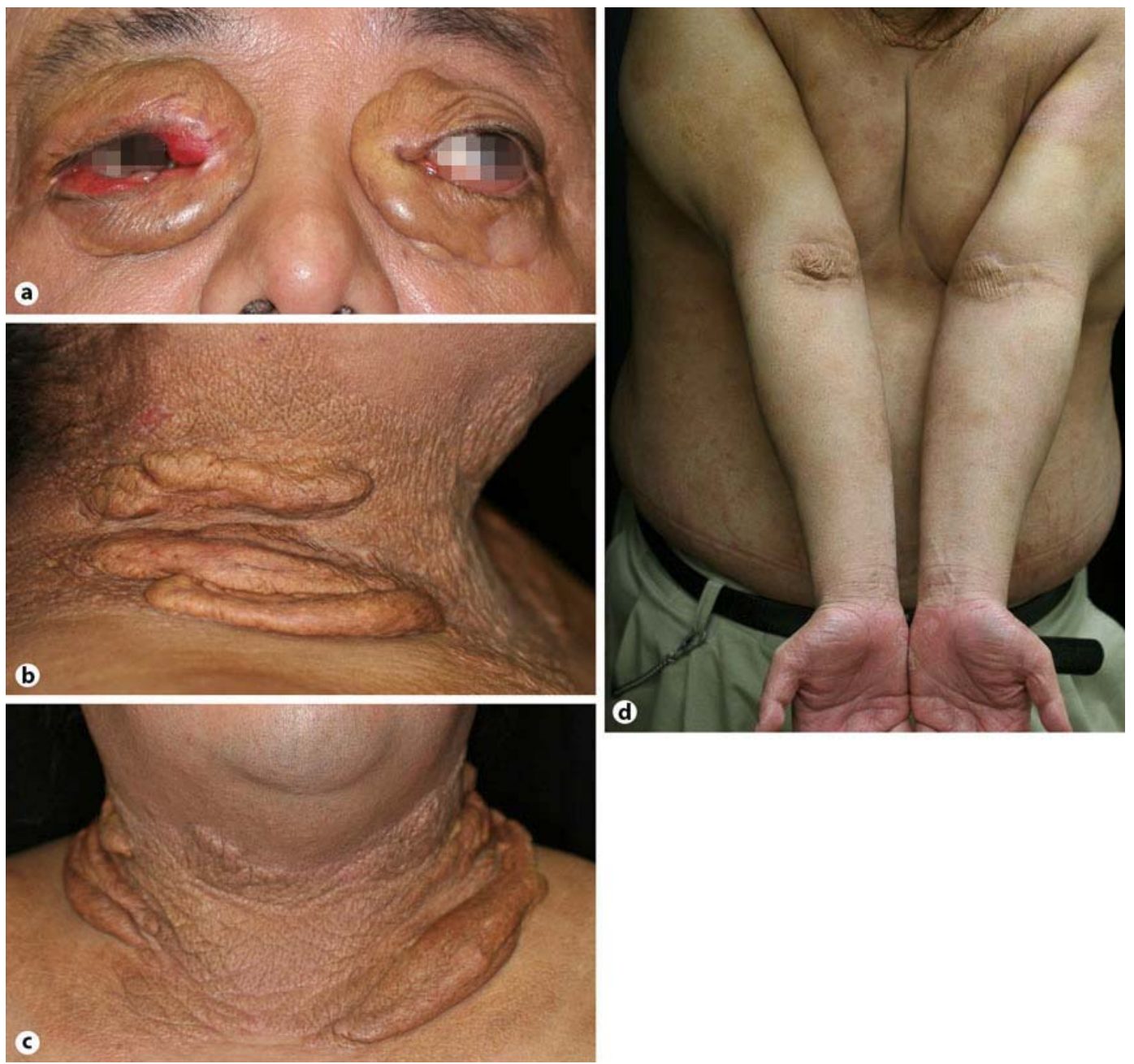

Fig. 1. a Ring-like yellowish tumors in the periorbital regions. b, c Rope necklace-like yellowish tumors on the patient's neck. d Spindle-shaped yellowish tumors on the patient's cubital fossas and diffuse erythema and pigmentation on the patient's forearm. 

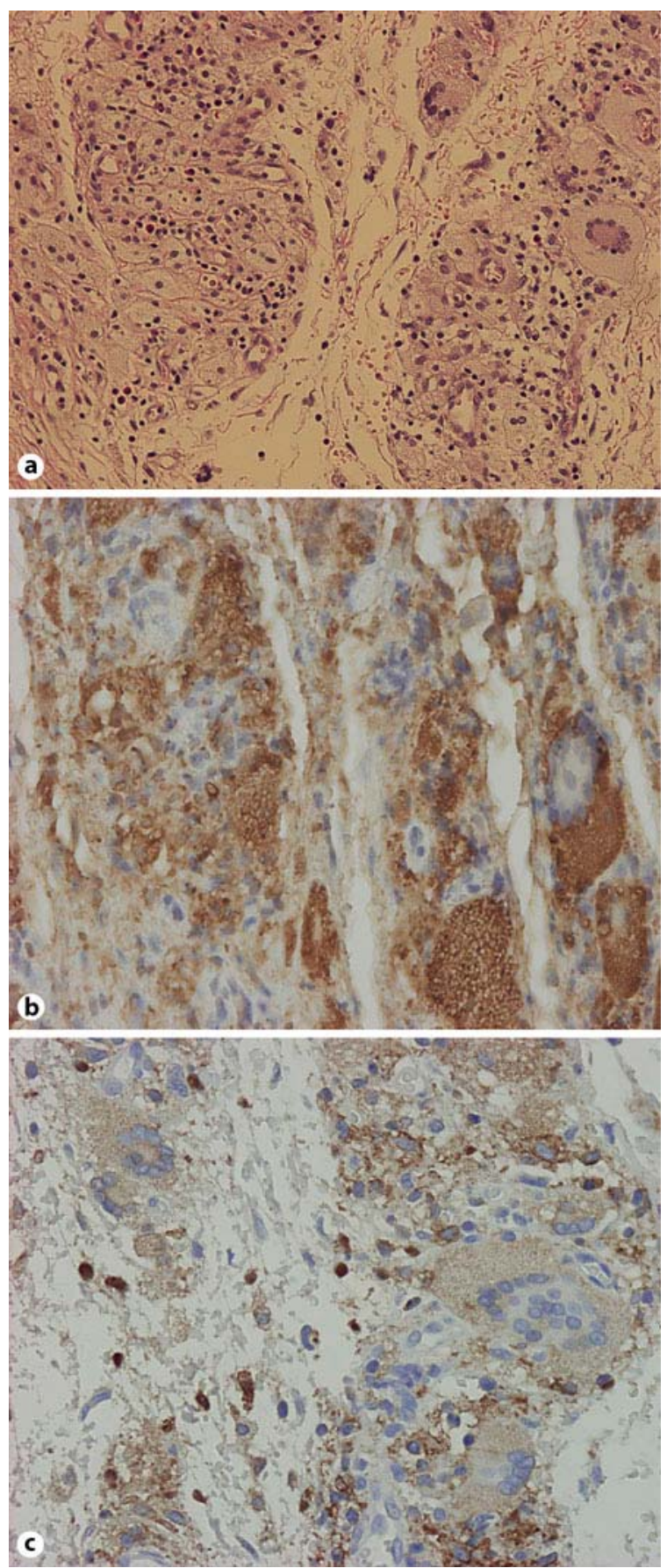

Fig. 2. Histopathological images from hematoxylin-eosin (a), original magnification, $100 \times$, and immunohistochemical analyses showing the expression of CD68 (b) and CD163 (c), original magnification, $200 \times$. 


\section{References}

1 Khamseh ME, Mollanai S, Hashemi F, Rezaizadeh A, Azizi F: Erdheim-Chester syndrome, presenting as hypogonadotropic hypogonadism and diabetes insipidus. J Endocrinol Invest 2002;25:727-729.

2 Tan AP, Tan LK, Choo IH: Erdheim-Chester disease involving breast and muscle: imaging findings. AJR Am J Roentgenol 1995;164:1115-1117.

-3 Garg T, Chander R, Gupta T, Mendiratta V, Jain M: Erdheim-Chester disease with cutaneous features in an Indian patient. Skinmed 2008;7:103-106.

$\checkmark 4$ Pertuiset E, Laredo JD, Liote F, Wassef M, Jagueux M, Kuntz D: [Erdheim-Chester disease: report of a case, review of the literature and discussion of the relation to Langerhans-cell histiocytosis]. Rev Rhum Ed Fr 1993;60:601-609.

5 Veyssier-Belot C, Cacoub P, Caparros-Lefebvre D, Wechsler J, Brun B, Remy M, Wallaert B, Petit H, Grimaldi A, Wechsler B, Godeau P: Erdheim-Chester disease. Clinical and radiologic characteristics of 59 cases. Medicine (Baltimore) 1996;75:157-169.

6 Ginhoux F, Tacke F, Angeli V, Bogunovic M, Loubeau M, Dai XM, Stanley ER, Randolph GJ, Merad M: Langerhans cells arise from monocytes in vivo. Nat Immunol 2006;7:265-273.

7 Brower AC, Worsham GF, Dudley AH: Erdheim-Chester disease: A distinct lipoidosis or part of the spectrum of histiocytosis? Radiology 1984;151:35-38.

$>8$ Waite RJ, Doherty PW, Liepman M, Woda B: Langerhans cell histiocytosis with the radiographic findings of Erdheim-Chester disease. AJR Am J Roentgenol 1988;150:869-871.

-9 Kenn W, Eck M, Allolio B, Jakob F, Illg A, Marx A, Mueller-Hermelink HK, Hahn D: Erdheim-Chester disease: Evidence for a disease entity different from Langerhans cell histiocytosis? Three cases with detailed radiological and immunohistochemical analysis. Hum Pathol 2000;31:734-739.

10 Furmanczyk PS, Bruckner JD, Gillespy T 3rd, Rubin BP: An unusual case of Erdheim-Chester disease with features of Langerhans cell histiocytosis. Skeletal Radiol 2007;36:885-889.

11 Schmidt HH, Gregg RE, Shamburek R, Brewer BH Jr, Zech LA: Erdheim-Chester disease: Low low-density lipoprotein levels due to rapid catabolism. Metabolism 1997;46:1215-1219.

12 Murota H, Bae S, Hamasaki Y, Maruyama R, Katayama I: Emedastine difumarate inhibits histamine-induced collagen synthesis in dermal fibroblasts. J Investig Allergol Clin Immunol 2008;18:245-252. 